\title{
Facet Machining of Silica Waveguides with Nanoscale Roughness without Polishing or Lapping
}

\author{
Lewis G. Carpenter, Helen L. Rogers, Christopher Holmes, James C. Gates and Peter G. R. Smith \\ Optoelectronics Research Centre, University of Southampton, Southampton, UK
}

To achieve low-loss free space coupling for integrated optics, device facets need to be smooth, free of chips and flat. The typical route for accomplishing these requirements is by traditional lapping and polishing. We report that high quality optical quality facets with a $S_{a}=4.9 \mathrm{~nm}$ can be machined using a simple dicing technique. In order to directly measure the scatter loss a device with a series of Bragg gratings is used to characterise the average interface loss per facet.

Traditional lapping and polishing of planar integrated optics is time consuming due to the multiple tasks required to perform the process, i.e. sample stacking and bonding, plate preparation, which all require constant human attention to ensure good facet quality. Created for the semiconductor industry, precision dicing saws are normally used to separate ICs from wafers but can be used to dice optical grade surfaces into materials such as silica. Dicing has been used to create such photonic structures as silica microcantilevers [1], multimode interference devices in silica flat fibre [2] and waveguides in lithium niobate [3]. Compared to traditional techniques, dicing offers automated wafer scale facet preparation requiring seconds to machine each facet.

We optimised dicing machining parameters to obtain optical quality facets in flame hydrolysis deposition silica-on-silicon. We focused on changing the feed rate and blade rotational speed while keeping constant the blade type (i.e. diamond size, diamond concentration and diamond bond type) and depth of cut. The diced silica surface roughness was analysed using a white light interferometer (Zemetrics, ZeScope). The smoothest silica facets produced had a three dimensional (areal), average surface $\left(\mathrm{S}_{\mathrm{a}}\right)$ of $4.9 \mathrm{~nm}$, as shown in Fig. 1 a). The optimised machining parameters for machining smooth silica facets, was found to be $<0.4 \mu \mathrm{m} / \mathrm{rev}$ using a diamond impregnated nickel bonded blade.
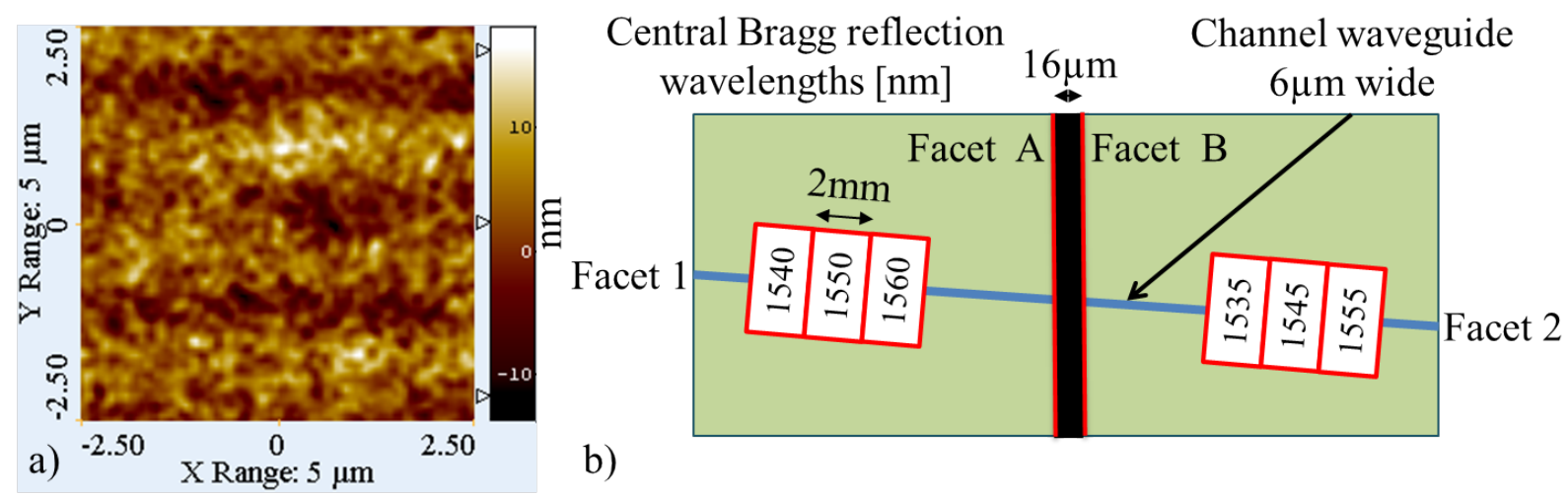

Fig. 1 a) a white light interferometer micrograph of a diced silica facet with $4.9 \mathrm{~nm}$ (Sa) of average surface roughness. b) is the chip schematic shown which includes waveguides and Bragg gratings to interrogate the average interface loss of facets $\mathrm{A}$ and $\mathrm{B}$.

To experimentally investigate the proportion of loss caused by scattering, as a consequence of facet surface roughness, a device containing a series of Bragg gratings was fabricated to allow a precise ratiometric Bragg grating loss measurement technique to be used. The integrated device, shown in Fig. 1 b), is realised by combing a groove, diced using the optimised machining parameters, with a single mode channel waveguides containing several Bragg gratings. To analysis the interface loss the reflection spectra is collected through both facets $(1$ and 2, Fig. 1 b)) of the device. By taking the ratio of the reflectivity of these Bragg gratings the total loss of the groove can be precisely determined. Subtracting the losses due to diffraction and Fresnel loss provides the individual average interface loss per facet (A and B, Fig. 1 b)). We will present the latest machining and interface loss results of silica facets created using precision dicing.

\section{References}

[1] L. G. Carpenter, C. Holmes, H. L. Rogers, P. G. R. Smith, and J. C. Gates, "Integrated optic glass microcantilevers with Bragg grating interrogation," Optics Express 18, 23296-23301 (2010).

[2] S. Ambran, C. Holmes, J. C. Gates, A. S. Webb, S. Member, L. G. Carpenter, F. Rafiq, M. Adikan, P. G. R. Smith, and J. K. Sahu, "Fabrication of a Multimode Interference Device in a Low-Loss Flat-Fiber Platform Using Physical Micromachining Technique," 30, 2870 2875 (2012).

[3] N. Courjal, B. Guichardaz, G. Ulliac, J.-Y. Rauch, B. Sadani, H.-H. Lu, and M.-P. Bernal, "High aspect ratio lithium niobate ridge waveguides fabricated by optical grade dicing," Journal of Physics D: Applied Physics 44, 305101 (2011). 